\title{
Lexis
}

Journal in English Lexicology

9 | 2015

Utterer-Centered Studies on Lexical Issues

\section{Lexique/grammaire. Le cas du khmer}

\section{Denis Paillard}

\section{(2) OpenEdition \\ Journals}

Édition électronique

URL : http://journals.openedition.org/lexis/931

DOI : 10.4000/lexis.931

ISSN : 1951-6215

\section{Éditeur}

Université Jean Moulin - Lyon 3

\section{Référence électronique}

Denis Paillard, « Lexique/grammaire. Le cas du khmer », Lexis [En ligne], 9 | 2015, mis en ligne le 13 mai 2015, consulté le 21 avril 2019. URL : http://journals.openedition.org/lexis/931 ; DOI : 10.4000/ lexis.931

\section{@ $(\mathcal{\Theta \Theta}$}

Lexis is licensed under a Creative Commons Attribution-NonCommercial-NoDerivatives 4.0 International License. 


\section{Lexique/grammaire. Le cas du khmer}

Denis Paillard ${ }^{1}$

\section{Résumé}

Cet article questionne la distinction entre le lexique et la grammaire avec l'étude de quatre unités de la langue khmère qui ont chacune des emplois dits lexicaux et des emplois dits grammaticaux (un phénomène très massif tant en khmer que dans de nombreuses langues du monde). L'étude de ces quatre unités met en évidence le fait que chacune se définit comme ayant sa propre "grammaire » au sens où chaque emploi peut être défini par un ensemble de propriétés (une combinatoire) sans que l'on puisse désigner un de ces emplois comme premier. Cette approche remet en cause l'association souvent faite entre le grammatical et ce qui est « obligatoire».

Dans les langues indo-européennes où la distinction lexique/grammaire a une visibilité empirique avec l'existence de classes ouvertes (le lexique) et de classes fermées (les mots grammaticaux) la notion de grammaticalisation est utilisée pour décrire le réemploi grammatical de telle ou telle unité du lexique ${ }^{2}$. En l'absence de tels inventaires stabilisés en khmer, certains auteurs en concluent à l'inexistence dans cette langue des catégories grammaticales comme le temps, l'aspect, la diathèse, le nombre, etc. Tout ne serait selon eux qu'une affaire de grammaticalisation - le grammatical n'existant que par défaut.

A l'opposé de cette approche, l'article défend la thèse selon laquelle l'identité d'une unité ne saurait être définie que par sa variation - ce terme de variation recouvre les notions de polysémie et de polycatégorialité souvent utilisées pour définir les différents emplois d'une unité, mais elle ne reprend pas la distinction entre le lexical et le grammatical. Cette analyse débouche sur une réflexion plus générale sur le statut des catégories (temps, aspect, diathèse, nombre, etc.) et leur mode de définition.

Selon les unités en jeu, chaque langue compte tenu des formes en jeu peut être considérée comme présentant une réalisation particulière de telle ou telle catégorie (on retrouve ici la notion de chréode introduite par Antoine Culioli). Prendre en compte la très grande diversité des réalisations possibles contribue en retour à un enrichissement de la réflexion sur le statut des catégories. D'où un double mouvement : des catégories vers les formes/les marqueurs, d'une part, des formes/des marqueurs dans une langue donnée vers les catégories, d'autre part.

Mots-clés : khmer - lexique - grammaire - catégorie grammaticale - identité sémantique - variation

\footnotetext{
${ }^{1}$ Université Paris Diderot. Laboratoire de linguistique formelle (UMR 7110) : denis.paillard@linguist.univparis-diderot.fr

${ }^{2}$ Cela ne signifie pas que la distinction soit absolue. Et de notre point de vue on ne peut pas fonder théoriquement la distinction lexique / grammaire y compris pour les langues indo-européennes.
} 


\begin{abstract}
This article questions the distinction between grammar and the lexicon through a study of four Khmer units, each of them presenting both lexical and grammatical uses, as can be observed in many languages in the world. The distinction between what is lexical and what is grammatical is highly questionable as each unit turns out to have its own distribution and its own combinatory and syntactic constraints. Besides, it is impossible to single out a particular use which could be held as specifically basic.

In Indo-European languages, the lexicon/grammar distinction has a certain visibility since two sets of units can be opposed easily enough: an open set, that of the lexicon, and a closed one, that of grammatical units. The use of lexical units for grammatical purposes thus seems to justify the use of the notion of "grammaticalization". But such an inventory proves impossible in Khmer, where categories such as tense, aspect, diathesis, number, etc. do not exist.

This article argues that what makes a word's identity is its very variation, not grammaticalization. Variation is to be understood both in terms of polysemy and of grammatical classification (polycategoriality), all the more so as, as a general rule, a Khmer unit is not linked to one stable category. This leads us to question the very foundations of what is apprehended as a grammatical category.

Any language uses units which, in their diversity, can be held as specific representatives of a given category (an illustration of the notion of chreod put forward by Antoine Culioli). Taking into account the various realizations of this category just as they can be observed contributes to deepen the reflection about its foundations. Hence a double movement: from the categories to the forms/markers, and from the forms/markers of a given language to the categories.
\end{abstract}

Keywords: Khmer - lexicon - grammar - grammatical category - word identity variation

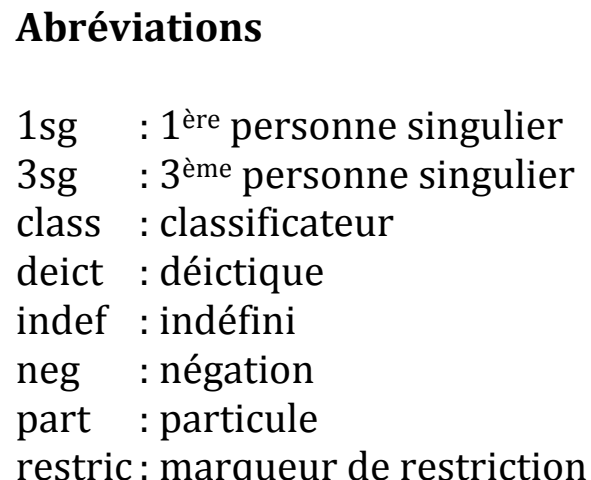




\section{Introduction}

La distinction entre unités lexicales et unités grammaticales a une visibilité empirique dans des langues comme le français et l'anglais mais tel n'est pas le cas de nombreuses langues, et en particulier des langues d'Asie du Sud-Est. L'objet de cet article est de reprendre la question de la distinction grammaire/lexique en référence à la langue khmère. En rapport étroit avec la possible distinction entre unités lexicales et unités grammaticales se pose la question du fondement des catégories comme nombre, détermination, temporalité, aspect, diathèse, modalité, etc.

La distinction entre lexique et grammaire - et plus généralement entre le lexical et le grammatical - repose souvent sur deux critères :

- Les unités grammaticales appartiennent à des listes fermées, alors que les unités lexicales entrent dans des listes ouvertes ;

- Le grammatical serait «obligatoire», position défendue en particulier par Jakobson [1963: 197, 201] :

Le caractère obligatoire des catégories grammaticales est le trait spécifique qui les distingue des significations lexicales [...] La grammaire est un véritable ars obligatoria comme disaient les scolastiques; elle impose au locuteur des décisions par oui ou non.

La frontière entre lexique (le lexical) / grammaire (le grammatical) telle qu'elle apparaît dans la majorité des langues indo-européennes tend à être érigée en modèle pour les langues appartenant à d'autres familles: le grammatical serait ce qui est grammatical dans les langues indo-européennes (en premier lieu en anglais) et le lexical serait ce qui relève du lexique dans ces mêmes langues. L'ouvrage World lexicon of grammaticalization de Heine et Kuteva [2002] est un exemple particulièrement frappant d'une entreprise consistant à prendre le lexique de l'anglais comme lexique de référence. Mais paradoxalement, les exemples donnés dans ce livre témoignent de la fragilité d'une telle distinction et de l'impossibilité de lui donner un statut théorique. La notion même de grammaticalisation en tant que signifiant l'acquisition de significations grammaticales par une unité lexicale témoigne de la véritable porosité entre ce que l'on désigne comme le lexical et le grammatical.

De plus, la question n'est pas limitée aux seules catégories, comme le montrent les deux séries d'exemples où la même notion peut être exprimée par une unité lexicale (anglais, français), par un préfixe attaché à une base (russe) ou encore par une construction verbale en série (khmer) :

\begin{tabular}{|c|c|c|}
\hline \multicolumn{3}{|c|}{ Français, Anglais : tuer to kill } \\
\hline Russe : & $u$ (préfixe) - bit’ (« frapper ») & \\
\hline Khmer : & $\begin{array}{ll}\text { koat } & \text { day } \\
\text { 3sg } & \text { frapper3sg }\end{array}$ & $\begin{array}{l}\text { vie } \text { goap } \\
\text { mourir }\end{array}$ \\
\hline ança & trouver to find & \\
\hline Russe : & na (préfixe) idti (« aller») & \\
\hline Khmer : & $\begin{array}{l}\operatorname{rook}(\text { «chercher } ») \\
\operatorname{rook}(\text { ( chercher })\end{array}$ & $\begin{array}{l}\text { aan (" acquérir ») } \\
\text { həə⿱ (« voir ») }\end{array}$ \\
\hline
\end{tabular}


En même temps, l'association du grammatical à de l'obligatoire reste aujourd'hui encore une grille de lecture pour des langues comme les langues d'Asie du Sud-Est comme en témoigne cette citation de Bisang [2009] :

In a large number of languages in East and mainland Southeast Asia, grammaticalization is characterized by the following characteristics:

- Lack of obligatory categories and predominance of pragmatic inference even in the case of highly abstract grammatical concepts such as tense and or definiteness;

- Existence of rigid syntactic patterns (word-order patterns);

- No or limited coevolution of form and meaning.

The definition of obligatoriness adopted in this paper is that of Lehmann [1995]. A category is obligatory if the speaker is forced to specify that category by selecting a marker that belongs to it. [...]

The lack of obligatoriness is particularly remarkable in cases where the concept inferred is an abstract grammatical concept that is expressed by obligatory categories in Indo-European languages. While these functions are conventionalized in Indo-European, they are the product of pragmatic inference in many markers of East and mainland Southeast Asian languages. This is corroborated by the fact that in a number of cases one and the same marker may express different grammatical concepts in different situations or in different constructions.

Cette citation montre une fois encore que dans ces langues la distinction entre le grammatical et le lexical ne renvoie pas à deux listes d'unités, les unes lexicales, les autres grammaticales ; bien plus, elle met en évidence le fait qu'une même unité selon le contexte peut exprimer different grammatical concepts. En même temps, la notion d'inférence pragmatique mise en jeu par Bisang pour rendre compte des valeurs grammaticales de certaines unités rejoue/maintient la distinction entre le lexical et le grammatical.

Dans cet article, en référence à quatre unités du khmer, nous défendons la position que l'identité sémantique d'une unité est indépendante de la distinction entre valeur(s) lexicale(s)/valeur(s) grammaticale(s) - ce qui revient à ne pas recourir à la notion de grammaticalisation. L'identité d'une unité est définie par sa variation et en retour la variation est contenue dans cette identité ${ }^{1}$.

Chacune de ces unités soulève des questions différentes concernant la variation. Dans le cas de la première unité 7aoy ( «donner ${ }^{2}$ ) la variation est en premier lieu celle des constructions : on distinguera dix emplois nettement distincts (on verra que ?aoy a le statut de métaprédicat). Avec trəv («toucher accidentellement») la variation renvoie à un ensemble de valeurs apparemment profondément disparates : déontique nécessaire, conformité, détrimentalité, diathèse passive. Avec baan ("obtenir») la variation soulève la question des catégories grammaticales, baan pouvant prendre deux valeurs, l'une modale («possibility»), l'autre aspectuelle (« attainment $»^{3}$ ). Enfin, dans le cas de daoy, la variation pose de façon cruciale la question de la polycatégorialité, cette

\footnotetext{
1 Le terme de variation recouvre en fait les phénomènes souvent traités en termes de polysémie mais aussi de polycatégorialité. Le choix d'un terme unique vise à ne pas poser au départ la distinction entre le lexical et le grammatical.

2 Pour ?aoy, trav et baan nous donnons la traduction donnée par les dictionnaires khmer/français, mais cela ne présume en rien de leur identité sémantique comme on le verra ci-dessous.

${ }^{3}$ Nous reprenons ici la terminologie utilisée par Enfield [2003].
} 
unité étant définie comme nom, verbe, préposition, particule adverbiale et conjonction de subordination ${ }^{4}$.

\section{A propos de ?aoy}

Pour décrire les différents emplois de Paoy (10 au total), nous ne partirons pas d'une caractérisation sémantique de 7aoy, mais d'une définition de ?aoy comme métaprédicat qui met en relation deux événements E1 et E2, ce que nous noterons E17aoy E2, où E2 est l'événement principal, E1 étant le déclencheur de E2. Cette relation entre E1 et E2 a une dimension causative, mais nous verrons qu'il n'est pas possible de la ramener à une simple relation causale : E2 a une autonomie par rapport à E1 au sens où il est introduit indépendamment de E1, même si la validation de E2 est conditionnée par la prise en compte de E1. E1 et E2 correspondent l'un et l'autre à une relation prédicative que, sous une forme minimale ${ }^{5}$, nous noterons $\mathbf{a}_{1} \mathbf{r}_{1} \mathbf{b}_{1}$ (E1) et $\mathbf{a}_{2} \mathbf{r}_{2} \mathbf{b}_{2}$ (E2), ce qui donne :

\section{$\left(X \mathbf{Y}\right.$ Z) $\mathbf{a}_{1} \mathbf{r}_{1} \mathbf{b}_{1}$ ?aoy $\mathbf{a}_{2} \mathbf{r}_{2} \mathbf{b}_{2}(X \mathbf{Y} \mathbf{Z})^{6}$}

Chaque emploi/valeur de 7aoy correspond à une réalisation particulière de ce schéma abstrait: selon les cas, les différents éléments des deux relations prédicatives peuvent ou non être lexicalisés.

\subsection{Transfert}

La valeur de transfert est celle où le schéma a une réalisation minimale ; Paoy est le seul élément prédicatif. E1 se ramène à la prise en compte d'un agent, E2 à celle d'un bénéficiaire et d'un objet (cf. (1a)). Mais après 7aoy, il est possible d'avoir le verbe $t \dot{+} v$ « aller » (cf. (1b)) : bénéficiaire et objet sont les arguments du verbe $t \dot{t} v$. L'interprétation de (1a) et de (1b) n'est pas la même : dans (1a) il s'agit du salaire versé à chaque ouvrier, dans (1b) il s'agit des dépenses en main d'œuvre engagées par l'entrepreneur.

\begin{tabular}{|c|c|c|c|c|c|c|c|}
\hline camp & & kaay & samnay & koat & ?aoy & $\varnothing$ & kaməka \\
\hline тиәу & tyay & concernant & travail construction & $3 s g$ & ?aoy & $\varnothing$ & travailleur \\
\hline un & jour & & & & & & \\
\hline pram & rכבע & riel & & & & & \\
\hline $\operatorname{cinq}$ & cent & riel & & & & & \\
\hline
\end{tabular}

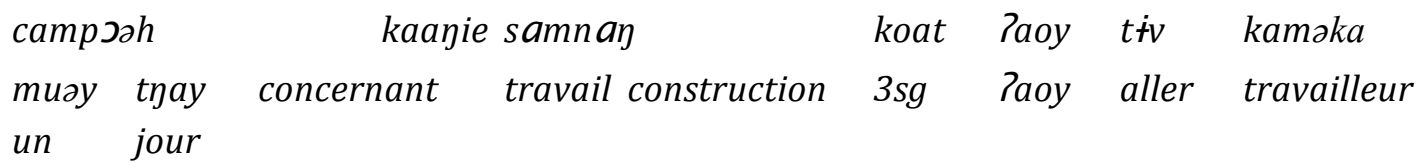

\footnotetext{
4 Chacune de ces unités ont fait l'objet d'une ou plusieurs descriptions détaillées figurant dans la bibliographie. Ici nous ne retenons qu'une partie des données étudiées.

${ }^{5} \mathbf{r}_{1}$ et $\mathbf{r}_{2}$ désignent le prédicat, $\mathbf{a}_{1}, \mathbf{a}_{2}, \mathbf{b}_{1}, \mathbf{b}_{2}$ les arguments du prédicat. Les autres constituants de E1 et E2 seront notés XYZ : ils n'interviennent pas directement dans la caractérisation des différents emplois.

${ }^{6}$ La présence de ?aoy dans cette notation met en avant son statut de métaprédicat.
} 
pram roכy riel

cinq cent riel

«Pour les travaux de construction il a dépensé 500 riels pour les ouvriers par jour (dépenses pour les salaires) $»^{2}$

\subsection{Bénéfactif}

Dans ce second type d'emploi, E1 contient un verbe (ici « acheter »). Quant à E2, soit il se ramène à un $\mathrm{N}$ bénéficiaire, soit on a la présence, comme pour le transfert, du verbe $t \dot{t} v$, et dans ce cas, l'objet de E1 devient le sujet de $t \dot{\boldsymbol{t} v}$ :

\begin{tabular}{|c|c|c|c|}
\hline koat & sizvph+v & $(t \dot{t+v})$ & mdaay koat \\
\hline acheter & livre & (aller) mère & $3 s g$ \\
\hline
\end{tabular}

On a deux événements : l'achat du livre (E1) et le livre est destiné à la mère (E2).

\subsection{Délégatif} sujet :

Par délégatif il faut entendre le fait qu'un sujet valide un procès à la place d'un autre

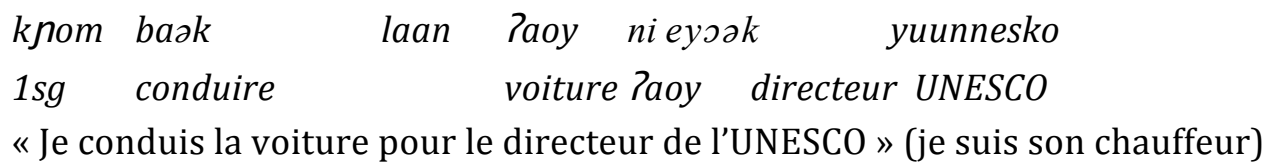

Dans ce cas, E1 est un événement, E2 se limite à un $\mathrm{N}$ qui, en l'absence de $\mathrm{V}$ en $\mathrm{E} 2$, s'interprète comme le sujet du même verbe que dans E1.

\subsection{Construction $\mathrm{N}$ kuə Paoy V}

Dans la construction $\mathbf{N}$ kuə ?aoy $\mathbf{V}$, kuə signifie que le $\mathbf{N}$ est adéquat pour vérifier une des places d'argument du V qui suit ?aoy:

(4) koat kuə ?aoy cang saəc

3sg kuə ?aoy vouloir rire

«Il est ridicule » (il donne envie de rire)

\subsection{Jussif}

7aoy peut être employé après un verbe exprimant une injonction et être suivi d'un prédicat qualitatif. En l'absence de Zaoy le prédicat qualitatif spécifie le procès. Avec ?aoy il s'interprète comme un objectif à atteindre en validant le procès visé, comparer :
(4a) $\begin{array}{ll}n \dot{+} \boldsymbol{v} & \varnothing \\ & \text { rester } \varnothing\end{array}$
sjizm
(sdap kee
niyiay san)
rester $\varnothing \quad$ tranquille
écouter $3 p l$ parler d'abord 
« Reste tranquille (écoute d'abord les autres)»

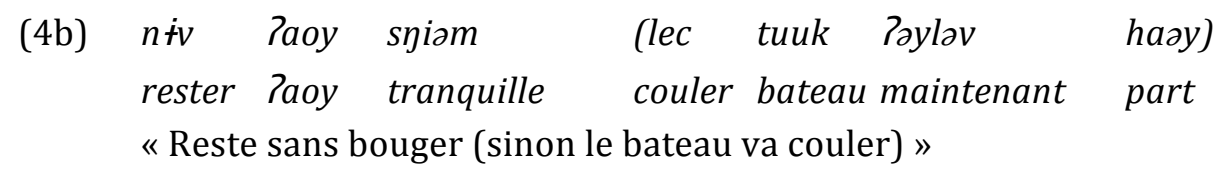

\subsection{Permissif}

Le permissif correspond aux cas où Paoy est suivi d'une séquence du type NV où le $\mathrm{N}$ désigne un sujet humain intéressé à la réalisation du procès exprimé par le $V$, procès qui, provisoirement, dépend d'un autre sujet, il peut s'agir du locuteur (ex. (5)) ou encore d'un sujet explicité à gauche de 7aoy (ex. (6)) :

(5) Paoy knom triv phaay

7aoy $1 \mathrm{sg}$ aller aussi

« Laisse-moi venir aussi »

(6) koat Paoy Daraa tiv

3sg Raoy Dara aller

«Il a laissé Dara venir avec lui »

\subsection{Causatif}

Le mécanisme est le même que pour le permissif à la différence près que le sujet du $\mathrm{V}$ suivant ?aoy a priori n'est pas intéressé par le procès. C'est le sujet (locuteur/interlocuteur, (7) ou $\mathrm{N}$ humain, (8)) placé devant 7aoy qui vise la réalisation du procès :

$$
\begin{aligned}
& \text { peel koat moכk dal Paoy koat cam knom bantec } \\
& \text { temps 3sg venir arriver ?aoy 3sg attendre } 1 \mathrm{sg} \text { un peu } \\
& \text { "Quand il arrivera, dis-lui de m'attendre un peu » }
\end{aligned}
$$

(8) koat ?aoy viз cam knom bantec

$3 s g$ ?aoy $3 s g$ attendre $1 s g$ un peu

«Il lui a dit de m'attendre un peu »

\subsection{Factitif}

C'est dans ce cas que le schéma est réalisé pleinement: E1 et E2 se présentent comme deux propositions, la première s'interprétant comme déclenchant la seconde. Le $\mathrm{V}$ dans $\mathrm{E} 1$ est soit un $\mathrm{V}$ ayant une dimension causative soit un verbum dicendi. On notera que dans ce cas la présence de Paoy n'est pas obligatoire, mais l'interprétation n'est pas la même ; comparer :

$\begin{array}{llllllrl}\text { knom } & \text { noam } & \text { koat } & \text { moगk } & \varnothing & \text { maəl } & \text { pteah } & \\ 1 \text { sg } & \text { conduire } & & 3 s g & \text { venir } & \varnothing & \text { regarder } & \text { maison }\end{array}$


«Je l'ai amené voir la maison" (pour qu'il voie comment je vis ou pour qu'il sache où j'habite)

$\begin{array}{lllllll}\text { knom noam } & \text { koat } & \text { mosk } & \text { ?aoy maวl pteah } & \\ 1 \mathrm{sg} & \text { conduire } & & 3 s g & \text { venir } & \text { ?aoy regarder maison }\end{array}$

«Je l'ai amené voir la maison » (la maison est en vente et il est un acheteur potentiel ou il doit être embauché comme gardien)

Dans (9) sans Paoy il s'agit d'un seul événement associant deux sujets alors que dans (10) il s'agit de deux événements distincts, le sujet du V dans E2 est intéressé à ce procès indépendamment du sujet de E1. Cette autonomie de E2 apparaît nettement dans (11) où le $\mathrm{V}$ de $\mathrm{E} 2$ est détrimental (dans ce cas, le sujet du V est obligatoirement après 7aoy, alors que dans (10), il est devant ?aoy) :

$\begin{array}{lllllll}\text { knom } & \text { soomtooh knom noam } & \text { ?aoy bay pi lba? } & \text { daoysaa } & \text { knom } \\ 1 \mathrm{sg} & \text { demanderpardon } & 1 \mathrm{sg} & \text { conduire } & \text { ?aoy } 2 \mathrm{sg} & \text { être difficile } & \grave{a}-\end{array}$

cause $1 \mathrm{sg}$

" Je vous demande pardon pour vous avoir mis dans une situation difficile »

\subsection{Optatif}

Paoy figure à l'initiale précédé ou non de la négation kom et suivi de tae marqueur de restriction. Dans une situation complexe (aux issues multiples) présente contextuellement 7aoy signifie que la seule chose qui compte est E2 ; dans (12) seul le premier laoy est concerné :

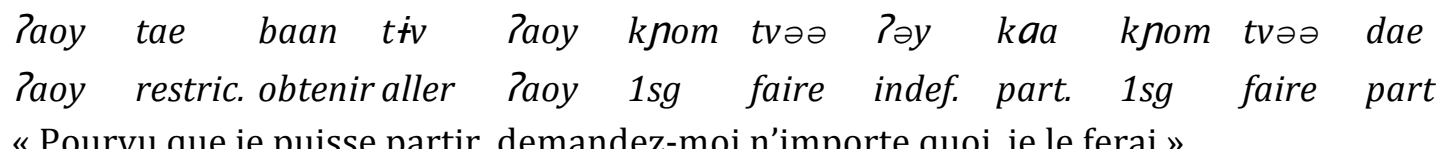

«Pourvu que je puisse partir, demandez-moi n'importe quoi, je le ferai »

\subsection{Critiquer/réprimander}

Combiné avec les verba dicendi thaa et sday, ?aoy signifie que le sujet qui suit 7aoy fait l'objet d'une critique ou d'une réprimande. L'objet de la critique ou de la réprimande est donné dans le contexte gauche mais n'est pas repris après laoy. De ce point de vue, «critiquer»/« réprimander» revient à désigner (verbalement) un sujet comme responsable d'un événement négatif :

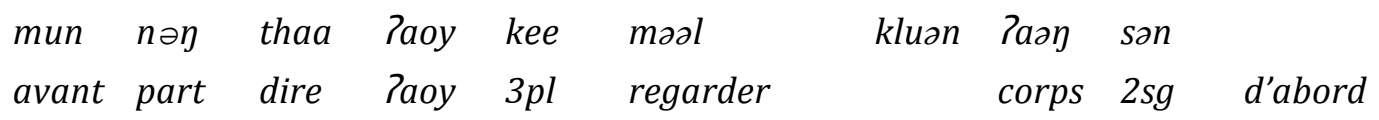

«Avant de critiquer les autres, regarde-toi d'abord» (à l'adresse de quelqu'un qui critique les autres pour leur tenue vestimentaire) 


$\begin{array}{cccccccc}\text { (14) bae } & \text { vie } & \text { moวk } & \text { yit } & \text { kom sday } & \text { ?aoy vie } \\ \text { si } & 3 s g & \text { venir tard } & \text { nég. } & \text { dire } & \text { ?aoy } & 3 s g\end{array}$

«S'il est en retard, ne le réprimande pas »

Synthèse. Les dix valeurs ci-dessus sont décrites chacune comme une réalisation particulière du schéma définissant laoy comme un métaprédicat mettant en relation deux événements E1 et E2 : la variation tient au fait que les composants des relations prédicatives de $\mathrm{E} 1$ et $\mathrm{E} 2$ sont selon les cas réalisés ou non.

\section{A propos de $\operatorname{trav}^{7}$}

En tant que verbe, trəv signifie « toucher accidentellement » comme dans l'exemple suivant :

(15a) koat vay trov kbaal knom

$3 s g$ frappertrov tête $1 s g$

«Il m'a heurté à la tête »

(15b) koat vay kbaal knom

$3 s g$ frappertête $1 s g$

" Il m'a frappé à la tête » (le coup est volontaire)

Dans (15b) où l'on a uniquement le verbe " frapper », le procès est présenté comme intentionnel, alors que dans (15a) le coup est présenté comme involontaire.

Nous proposons l'identité sémantique suivante pour trav : étant donné un élément e2 localisé/actualisé, un élément e1 se retrouve en contact avec e2. Du point de vue de la dynamique de l'événement c'est e1 qui est concerné. Si dans le cadre de cet événement e1 entre en contact avec e2, cette mise en relation n'est pas à l'initiative de e1 : du point de vue de l'événement impliquant e1, e2 est un élément contingent, a priori sans rapport à l'événement.

Indépendamment de sa valeur comme verbe, trav présente quatre grandes classes d'emploi : conformité, détrimental, diathèse passive et obligation-nécessité. Ci-dessous, nous montrerons à partir d'un petit nombre d'exemples comment ces quatre types d'emploi s'interprètent comme des réalisations particulières de cette identité de trəv.

\subsection{Conformité}

La valeur de conformité peut être illustrée par les exemples suivants :

koat trov cnaot

\footnotetext{
7 On retrouve dans d'autres langues d'Asie du Sud-Est le même verbe avec un ensemble comparable de valeurs. Pour le Mon, cf. Jenny [2003: 231] : «In modern Mon $t \varepsilon h$ has a number of translations, including "hit" (a mark, a target), "touch", "be correct", be cheap" as a full verb, "have to", "must", "undergo", PASSIVE (ADVERSATIVE) as preverbal auxiliary, and "correctly", "able", "inadvertently", "unintentionally" as postverbal auxiliary. » Dans cette citation on notera le recours systématique à la traduction pour distinguer les différents emplois.
} 
3sg trav loterie

"Il a gagné le gros lot»

mhoop nih trav moat knom nah!

plat deict trav bouche $1 \mathrm{sg}$ très

«J'aime beaucoup ce plat!»

$\begin{array}{llllll}\text { caacak kat caek nih trəv } & \text { taam } & \text { ytte?thoa haəy } & \\ \text { loup couper partager } & \text { deict. } & \text { trəv } & \text { suivre justice } & \text { part } \\ \text { "Le partage que le Loup a fait est juste } & & & & \end{array}$

Le terme "conformité » signifie que e2 est défini comme un pôle de référence donnant lieu à une valuation (en termes de conformité) de l'événement impliquant e1. On a d'un côté un événement actualisé (il se définit par son ancrage dans un espacetemps singulier), de l'autre, un pôle de référence dont la pertinence excède l'événement actualisé. La fonction de trəv est de mettre en relation l'événement mettant en jeu e1 avec le pôle de référence que représente e2.

Le terme pris comme pôle de référence e 2 a des statuts très variés, ce qui tend souvent à conférer à la séquence formée avec trəv une dimension idiomatique, encore soulignée par la traduction en français. Nous pensons qu'il est possible de distinguer au moins deux grands cas :

- le pôle de référence e 2 est un terme qui acquiert ce statut dans le cadre de sa mise en relation avec e1. Ce cas est de loin le plus fréquent et recouvre, nous l'avons dit, une très grande diversité.

Ainsi, dans (16) le billet ( $\mathrm{e} 1$ ) acheté par X possède le numéro correspondant au numéro défini comme le numéro gagnant lors du tirage (e2). Dans (17), le plat (e1) préparé avec tel et tel ingrédient se révèle être à mon goût (e2). Dans les deux cas il n'y a a priori pas de rapport entre e1 et e2 : e2 est défini indépendamment de e1.

- le pôle de référence est introduit par des marqueurs comme daoy "par/selon » et par taam "suivre/suivant ». Ce pôle de référence s'interprète comme une norme ou une règle ayant une existence indépendamment de l'événement particulier impliquant e1. Dans (18), l'événement que constitue le partage réalisé par le loup se révèle en accord avec les principes d'équité/justice qui définissent ce qu'est un bon partage.

\subsection{Détrimental}

Cette valeur est celle qui est la plus proche de la valeur de trav comme verbe :

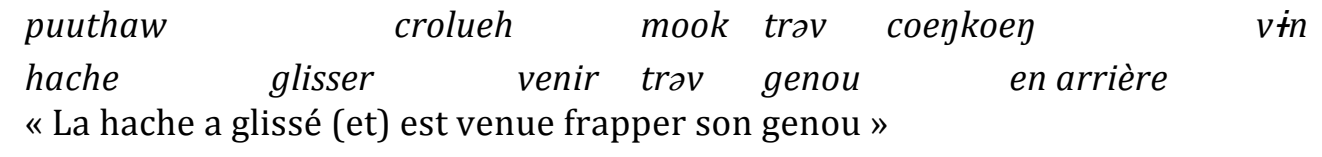

$\begin{array}{llll}\text { niey trav } & \text { snae } & \text { kee hasy } \\ \text { fille trav charme } & 3 s g & \text { part. }\end{array}$

"Quelqu'un a jeté un sort à cette fille »

$\begin{array}{lll}\text { monuh məneak trəv krəəh tha? } & \text { bok laan } \\ \text { personne une personne trəv accident } & \text { frappervoiture } \\ \text { «Une personne a eu un accident de voiture » } & \end{array}$


(22)

msglmgn knom trəv moat lav knom
hier
« Hier je me suis fait gronder par mon père ! "

Dans (19) e1 désigne un événement négatif qui affecte e2 (un individu). Dans (21) et (22), c'est e2 qui prend une dimension négative et un individu (e1) est mis en relation avec e2. Dans (23) le mécanisme est quelque peu différent et repose sur le fait que le fils (e1) se retrouve bien malgré lui en relation avec la bouche (c.à.d. le discours) de son père.

Ci-dessous nous donnons deux exemples mettant en jeu la valeur conformité et la valeur détrimentale. Dans l'exemple (23), le second locuteur joue sur le mot trav et passe de la valeur de conformité (déjà présente dans la question de $\mathrm{S}_{0}$ ) à la valeur détrimentale (on retrouve moat déjà présent dans (22) mais avec un autre statut) :

(23) $\left[S_{1}\right.$ vient remettre son travail au directeur; à la sortie, $S_{0}$ demande à $S_{1}$ si le directeur a apprécié le travail qu'il a remis]

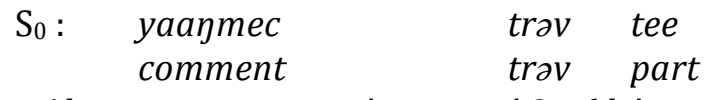

«Alors, comment ça s'est passé ? » (il était content ?).

$\mathrm{S}_{1}:$ trəv trəv moat laəy spək muk haәy trav trav bouche monter engourdi visage part

«Tellement content que je me suis fait engueuler comme du poisson pourri» (présence d'une pause entre les deux trəv).

Et l'exemple (24) ci-dessous, en fonction de la prosodie, peut recevoir une interprétation de type conformité (a) ou de type détrimental (b) :

$\begin{array}{lllll}\text { tvəə } & \text { ṫv } & \text { trəv } & \text { วəylav } & \text { haəy } \\ \text { faire } & \text { aller } & \text { trəv } & \text { maintenant } & \text { déjà }\end{array}$

a. "Continue, tu y es presque »

b. "Continue comme ça, et tu vas voir ce que tu vas prendre comme correction" (accent contrastif sur trav, qui signifie qu'il y a un enjeu intersubjectif : " continuer» $\left(\mathrm{S}_{1}\right)$ vs « ne pas continuer » (So)).

L'interprétation (a) est possible dans un contexte où un élève résout un problème de mathématiques et il approche de la solution. Son professeur l'encourage en utilisant l'énoncé (24). L'interprétation (b) met en scène une mère qui menace son fils qui s'entête à faire ce qu'elle lui interdit de faire.

\subsection{Nécessité - prédiction - besoin}

Cet emploi de trav regroupe trois valeurs proches : nécessité/obligation (ex. (25), (26)), prédiction (ex. (27)), besoin (ex. (28) et (29); dans le cas de " besoin », trav est suivi de kaa « affaire»).

\begin{tabular}{|c|c|c|c|c|c|c|}
\hline kлom & kmian & bay & knom & trav & $t \dot{t+w}$ & $p^{h} s a a$ \\
\hline & ne pas avoir & riz & $1 s g$ & $\operatorname{tr} \partial v$ & aller & march \\
\hline
\end{tabular}


(26)

\begin{tabular}{|c|c|c|c|c|c|c|}
\hline sraec ?aey & $\operatorname{tr} \partial v$ & $m כ$ kכ & сиәр & knom & knom & mien \\
\hline demain & $2 s g$ & $\operatorname{trav}$ & venir & rencontrer & $1 s g$ & $1 s g$ \\
\hline samk & & $\operatorname{trav}$ & niyəy & & & \\
\hline
\end{tabular}

$\begin{array}{lllllll}\text { taam leec bia } & \text { khae } & \text { kraoy loวk trav baan luy craən } \\ \text { suivant numéro } & \text { carte } & \text { mois après } & \text { monsieur } & \text { trav obtenir }\end{array}$
argent beaucoup

«D'après les numéros des cartes, le mois prochain vous devez gagner beaucoup d'argent»

$\begin{array}{lllllll}\text { knom trəv kaa } 20 \$ & \text { daəmbey } & \text { bang tlay } & \text { tik } \\ 1 \text { sg trəv affaire } 20 \$ & \text { pour } & \text { payer } & \text { prix } & \text { eau } \\ \text { "J'ai besoin de } 20 \$ \text { pour payer la facture d'eau » } & & \end{array}$

knom trəv kaa monuh pii neak сиәy tvəə kaa $1 \mathrm{sg}$ trov affaire personne deux class aider faire affaire «J'ai besoin de deux personnes pour m'aider dans le travail »

Ces trois types de données présentent les propriétés suivantes :

a. un sujet a priori humain ( $\mathrm{e} 1$ ) est confronté à la validation d'un procès ( $\mathrm{e} 2$ ) ;

b. la construction du procès ( $\mathrm{e} 2$ ) relève non pas du sujet mais d'une instance externe (explicitée ou non). Dans le cas de la nécessité, la prise en compte de e2 est liée à la validation d'un objectif que se fixe e 1 ; dans le cas de la prédiction, e2 est construit par un sujet autre que e1 (voyant, chamane, etc) ; dans le cas du besoin c'est un manque ressenti par le sujet e1 qui construit e2 ;

c. la validation effective du procès (e2) par le sujet (e1) est en attente et n'est pas garantie ;

d. la fonction de trav réside dans la mise en relation du sujet (e1) avec le procès (e2) construit indépendamment.

Les trois valeurs (nécessité/prédiction/besoin) se caractérisent par des différences d'ordre syntaxique et lexical. Elles peuvent être considérées comme correspondant à une pondération variable ${ }^{8}$ sur les éléments $\mathbf{e} \mathbf{1}$ et $\mathbf{e} 2$ mis en relation par trəv :

- nécessité : équipondération du sujet $(\mathbf{e} 1)$ et du procès (e2) qui possèdent l'un et l'autre leur propre visibilité ;

- prédiction : pondération sur le procès (e2) (le sujet est «dépossédé » de son destin par les cartes) ;

- besoin : pondération sur le sujet (e1) (le procès permettant de satisfaire le besoin n'est pas encore sélectionné).

\footnotetext{
${ }^{8}$ Par pondération variable, il faut entendre le fait que les deux éléments n'ont pas la même saillance : dans le cas du besoin et de la prédiction un seul des éléments a une réelle visibilité.
} 


\subsection{Diathèse passive}

Le schéma syntaxique régulier pour le passif est :

\section{(...) GN trav GN V (...)}

(30)

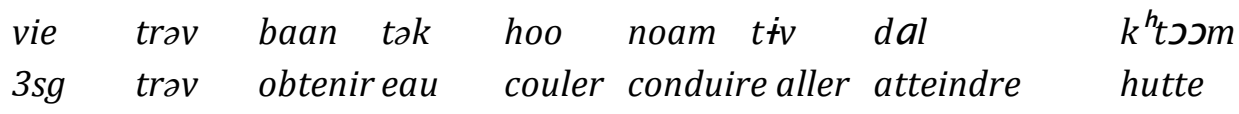

«Il fut emporté par le courant jusqu'à la hutte (du dieu) »

(31) Phnom Penh trav kmay vay baek thnay tii

Phnom Penh trav ennemifrappercasser date lieu

«Phnom Penh a été pris par l'ennemi tel jour»

conlməh trəv baan poolih cap dak kuk
criminel trəv obtenir police arrêter mettre prison
«Le criminel a été arrêté par la police » [Thach : 2011]

Dans le cas du passif, on retrouve le mécanisme décrit pour les trois premières valeurs. trəv inscrit un GN (e1) dans une relation prédicative GN V (e2) construite indépendamment de $\mathbf{e 1}$. L'instance de construction du procès n'est autre que l'agent du procès présent devant le verbe. GN $(\mathbf{e} 1)$ reçoit le statut d'objet du procès.

Synthèse : la description des cinq grands types d'emplois de trəv a permis de mettre en évidence ce qu'ils ont en commun : trəv a pour fonction d'inscrire un terme e1 dans l'espace d'un terme e2 construit indépendamment de $\mathbf{e} 1$. Cela est présent aussi bien dans la valeur «toucher » de trav que dans les quatre autres valeurs. De ce point de vue, il n'y a pas lieu de considérer que l'emploi de trəv dans les valeurs "conformité », « détrimental », "nécessité » et " passif » correspondent à une désémantisation de trəv signifiant "toucher accidentellement»: ce qui varie d'un emploi à l'autre, ce sont les propriétés syntaxiques et sémantiques des termes $\mathbf{e} \mathbf{1}$ et $\mathbf{e 2}$, qui donnent un contenu différent à leur mise en relation. Rien ne permet de considérer que l'emploi lexical de trav soit premier : il est une configuration parmi d'autres. Notre approche ne fait donc pas appel à la notion de grammaticalisation. Le fait de privilégier la valeur de trav comme verbe plein ne permet pas de dégager ce qui fonde l'unité du mot, sa cohérence du point de vue du système de la langue. Chaque emploi est à la fois contingent (rien ne permet $a$ priori de le prévoir) et nécessaire: il est régi par des propriétés syntaxiques et sémantiques spécifiques et régulières. Sur le plan syntaxique, trois des grandes valeurs peuvent être considérées comme des constructions verbales sérielles (CVS) ${ }^{9}$ où trəv apparaît comme V1 (« nécessité » et « passif ») ou comme V2 (« toucher »).

\section{A propos de baan}

Le verbe baan est le troisième verbe décrit. A la différence des deux verbes précédents, comme verbe unique il n'a que des emplois relativement restreints :

\footnotetext{
${ }^{9}$ A propos de baan, nous reviendrons sur la notion de CVS.
} 
$k^{h}$ गm baan koon proh

$1 s g$ baan enfant mâle

«J'ai un fils!» (un père à la clinique d'accouchement après qu'on lui a annoncé la naissance de son enfant)

$$
\begin{aligned}
& k^{h} n \supset m \text { baan sievp }{ }^{h} \partial \\
& 1 s g \text { baan livre } \\
& \text { "J'ai eu un livre » (à un tirage au sort) }
\end{aligned}
$$

Pour caractériser la sémantique de baan nous partirons de la formulation proposée par Enfield : "Khmer baan commonly means "come to have something" » [2003 : 168]. Dans les exemples (33)-(34), baan signifie qu'une entité (individu ou objet) se retrouve dans l'espace du sujet, sans que ce dernier soit à l'origine de cette acquisition. Comme le souligne Enfield, baan est " non agentif», ce que reflète l'impossibilité de l'avoir dans une injonction :

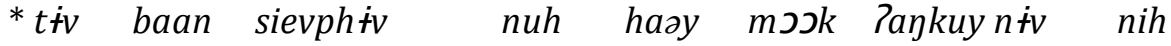

$$
\begin{aligned}
& \text { aller baan livre deict part. venir s'asseoir rester ici } \\
& \text { Litt. «Va et obtiens le livre, apporte-le et assieds-toi ici » }
\end{aligned}
$$

baan n'est également pas possible après le verbe trəv lorsque ce verbe marque l'obligation. Enfin il est incompatible avec des adverbes spécifiant le mode d'implication du sujet dans le procès.

Nous reformulerons la caractérisation proposée par Enfield en recourant à la notion de « chemin » qui fait directement écho à l'utilisation de come dans la définition de cet auteur. Mais alors que pour Enfield, la formulation « come to have » ne vaut que pour les emplois de baan comme verbe unique, nous chercherons à montrer ci-dessous que la formulation en termes de " chemin " permet de rendre compte des différents emplois de baan, comme verbe unique mais aussi dans les CVS.

Identité sémantique de baan: baan pose l'existence d'un chemin entre deux positions $\mathbf{e}_{1}$ et $\mathbf{e}_{2}: \mathbf{e}_{2}$ est la position de référence et $\mathbf{e}_{1}$ se présente comme une position d'extériorité première par rapport à $\mathbf{e}_{2}$. Selon le co-texte on privilégie $\mathbf{e}_{1}$ ou $\mathbf{e}_{2}$, mais les deux positions sont toujours prises en compte:

- s'il s'agit de la position de référence $\mathbf{e} 2$, le chemin est validé ;

- s'il s'agit de la position d'extériorité première $\mathbf{e} \mathbf{1}$ : le chemin est validable. 


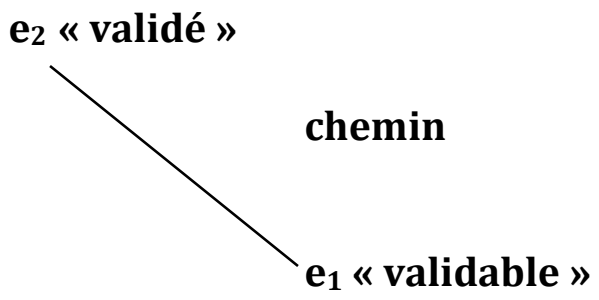

La notion centrale de chemin ${ }^{10}$ reliant deux positions apparaît clairement avec les emplois où baan joue le rôle de connecteur entre deux propositions $\mathbf{p}$ et $\mathbf{q}^{11}$. Dans ce cas il y a focalisation sur l'existence d'un chemin (correspondant à une mise en rapport) entre les deux propositions avec différentes interprétations possibles :

- q (est présenté comme la conséquence de $\mathbf{p}\left(\mathbf{e}_{1}\right)$ :

?aen vay vie nim(p)baan vie yum (q)

$2 s g$ frapper $3 s g$ deict baan $3 s g$ pleurer

«Tu l'as frappé (p), c'est pour ça qu'il pleure ! (q) »

- $\mathbf{p}\left(\mathbf{e}_{1}\right)$ a pour interprétation $\mathbf{q}\left(\mathbf{e}_{2}\right)$ :

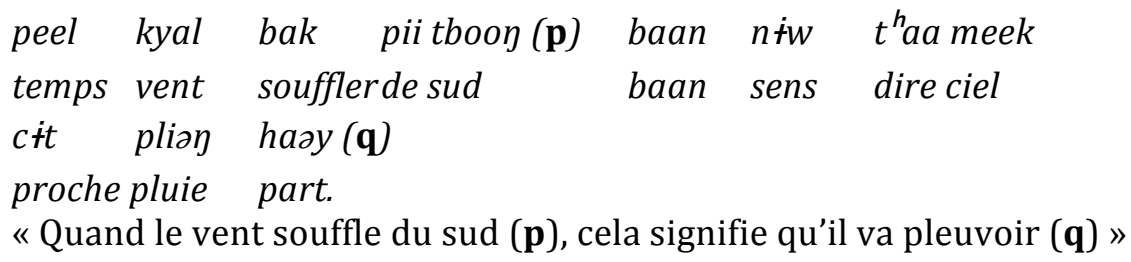

\section{1. baan dans les CVS}

Dans Paillard [2013c], nous proposons la définition suivante d'une CVS :

- $\quad$ une CVS est une suite de $V$ de la forme : $V_{1} \ldots\left(V_{i}\right) \ldots V_{n}$ (en khmer une CVS peut comporter jusqu'à une dizaine de $\mathrm{V}$ ) ;

- cette suite de $\mathrm{V}$ construit un événement complexe, chaque $\mathrm{V}$ (à l'exception du dernier $V$, noté $V_{n}$ ) est un « sous-événement» dans le cadre de cet événement complexe ;

- l'ordre d'introduction des V est pertinent (cf. ci-dessous) ;

- le $V_{n}$ introduit une détermination spécifiant l'interprétation de l'événement complexe formé par la combinatoire $V_{1}\left(V_{2} V_{i}\right)$. Le $V_{n}$ n'est pas «extérieur» à l'événement complexe mais il remplit une fonction particulière ${ }^{12}$.

\footnotetext{
${ }^{10}$ La présence de come dans la caractérisation de Enfield souligne le fait que to have ( $\mathbf{e}_{2}$ ) résulte d'un parcours préalable : cet «en-deçà de $\mathbf{e}_{2}$ » que nous notons $\mathbf{e}_{1}$ est essentiel pour comprendre le fonctionnement de baan.

${ }^{11}$ Nous ne discuterons pas en détail de cet emploi de baan qui devra faire l'objet d'une étude spécifique. A noter toutefois l'alternance baan (ex. (37))/ baan cie où cie est la copule (ex. (44) dans la partie consacrée à daoy).

${ }^{12}$ Cette définition d'une CVS en khmer se distingue de la définition généralement admise sur au moins deux points: a. tout $\mathrm{V}$ dans une CVS doit être considéré comme un V (ni grammaticalisation ni lexicalisation) ; b. le dernier V d'une CVS a un statut spécifique. Dans Paillard [2013c], on trouve une explicitation de ces différences.
} 
Pour rendre compte de cette combinatoire, une CVS doit être décrite sur deux plans :

- construction de l'événement complexe par avancées successives (les sousévénements). Lorsque nous parlons d'avancées successives, nous ne faisons pas référence à une lecture temporelle de l'événement (un événement complexe n'est pas réductible à une succession dans le temps de sous-événements) mais à une temporalité de la prédication, telle qu'elle se met en place avec la combinatoire des différents verbes (de leurs scénarios correspondant à leur identité sémantique). Chaque $\mathrm{V}$ est considéré du point de vue de son rapport aux autres verbes ;

- la CVS en tant que produit de cette combinatoire : elle se présente sous la forme d'une relation prédicative avec ses différents arguments.

Comme V1, mais aussi comme Vn dans une CVS, baan présente deux valeurs nettement distinctes, décrites par Enfield comme possibility d'une part, attainment d'autre part. Compte tenu de ces deux valeurs, l'une a priori d'ordre modal, la seconde d'ordre aspectuel, il peut être tentant de décrire une partie des emplois de baan (en fait la majorité) en termes de grammaticalisation (baan devenant alors soit un marqueur modal soit un marqueur aspectuel). Une telle approche est celle de Enfield pour le khmer mais aussi pour les équivalents de baan dans d'autres langues d'Asie du Sud-Est (lao, vietnamien, hmong, etc.). Jenny [2005] défend la même approche pour la langue mon. Du point de vue de la caractérisation de baan que nous proposons, ces deux valeurs correspondent à l'actualisation soit de $\mathbf{e}_{\mathbf{1}}$ (possibility) soit de $\mathbf{e}_{2}$ (attainment).

On notera que ces deux valeurs sont attestées tant avec baan comme V1 qu'avec baan comme Vn.

baan est en position de V1:

- chemin validable $\mathbf{e}_{\mathbf{1}}$ (possibility) :

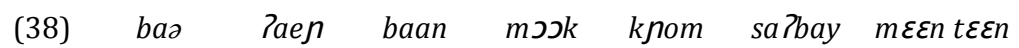

si $2 s g$ baan venir $1 s g$ heureux vraiment

«Si tu es en mesure de venir, je serai vraiment content»

- chemin validé $\mathbf{e}_{2}$ (attainment) :

(39) kfom baan riepkaa taam prapeynii $k^{h} m a e$

$1 \mathrm{sg}$ baan mariage suivre coutume khmère

« (Bien qu'en France) j'ai réussi à me marier selon la coutume khmère »

Comme le montre l'exemple (40), l'actualisation de $\mathbf{e}_{2}$ (chemin validé) ne désigne pas nécessairement un événement révolu (baan est V1) :

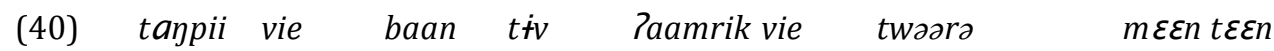

depuis $3 s g$ baan aller USA 3sg êtreodieux vraiment

a. « Depuis que son départ pour les Etats-Unis est acquis, il a une attitude vraiment odieuse » 
b. « Depuis qu'il a réussi à aller aux Etats-Unis, il a une attitude vraiment odieuse »

baan est en position de V2:

L'exemple (41) est compatible avec les deux interprétations :

(41) sdap ?an baan tee

écouter $1 \mathrm{sg}$ baan part

a. «As-tu compris ce que je t'ai dit?» (e2 : attainment)

b. « Est-ce que tu peux m'écouter ?» (e1 : possibility).

L'exemple (42) présente deux occurrences de baan la première comme V1, la seconde comme V2 (dans les deux cas on a la valeur de chemin validé) :

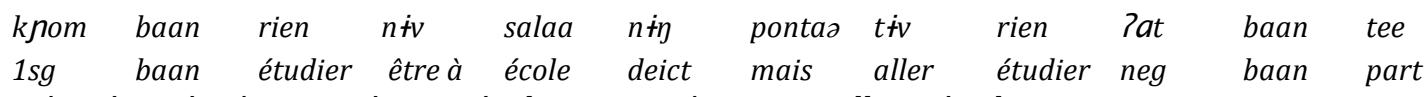

" J'ai réussi à m'inscrire à cette école mais je n'ai pas pu aller y étudier »

En position de V1, baan signifie qu'il existe un chemin qui conduit à un événement exprimé par le V2 $(\mathrm{Vn})$ : il s'agit de décrire la distance à parcourir ; selon qu'elle est à parcourir ou parcourue, l'interprétation de l'événement varie, possibilité dans un cas, réussite de l'autre.

En position de Vn, baan signifie que l'événement exprimé par le, ou les, $\mathrm{V}$ qui précèdent est identifié soit comme un chemin qui est à parcourir soit comme un chemin (déjà) parcouru. La notion de distance centrale dans le cas de baan en position de V1 n'est plus pertinente alors. Il y a stabilisation de l'événement soit en $\mathbf{e}_{1}$ soit en $\mathbf{e}_{2}$.

\section{Le cas de daoy}

Associé au cognate mon-khmer $t_{1} u y$ («to follow») dans A Mon-khmer comparative dictionary [2006], daoy est défini dans le dictionnaire khmer-anglais de Headley comme a. predicate "attached to, to follow, to obey" as well as "to love intimately, sexually"; $\mathbf{b}$. preposition "by, with, along"; c. particle : indicator of an adverb: "in a .... manner"; d. conjunction "because"13. Cette diversité des statuts syntaxiques attribué par Headley à daoy est en fait largement influencée par la traduction en anglais des différents types d'énoncés où daoy apparaît. De plus, une série d'emplois échappent à cette répartition catégorielle. On notera qu'en khmer préangkorien et angkorien [Jenner 2009a et b], daoy présente la plus grande partie de ses emplois actuels.

Ci-dessous nous proposerons une caractérisation sémantique pour daoy qui permet de rendre compte de la diversité de ses emplois, chaque emploi correspond à des constructions différentes - ce qui revient à poser que daoy possède sa propre grammaire, définie à partir du schéma suivant :

$$
\mathbf{X} \text { daoy } \mathbf{Y}
$$

\footnotetext{
${ }^{13}$ A cette liste, le Dictionnaire de l'Institut Bouddhique ajoute celui de «nom ».
} 
où $\mathrm{X}$ désigne une relation prédicative et $\mathrm{Y}$ est une séquence (de nature variable) dont le, ou les, constituants n'ont pas de statut dans la relation prédicative X. daoy signifie que la prise en compte de Y (re)définit l'interprétation de X.

En référence à cette hypothèse nous analyserons une série (limitée ${ }^{14}$ ) d'emplois de daoy.

koon nih daoy damboonmien nah
enfant ce daoy conseils très
"Cet enfant est très obéissant »

En (43), X se ramène au GN « cet enfant », et on pourrait être tenté de conférer à daoy le statut de verbe. L'existence à côté de (43) d'un énoncé proche avec le verbe taam (« suivre ») montre qu'il n'en est rien :
koon nih taam damboonmien nah
enfant ce taam conseils très
"Cet enfant suit les conseils (qu'on lui donne) »

Le statut de $\mathrm{V}$ de taam est confirmé par le fait qu'il est compatible avec la négation, ce qui n'est pas possible avec daoy:

(43b) koon nih min taam damboonmien

enfant ce nég taam conseils

"Cet enfant ne suit pas les conseils (qu'on lui donne)»

L'exemple (44) est comparable à (43) à la différence près que Y présent dans le contexte gauche n'est pas repris après :
koat mien luy craən
baan cie
srəy daoy
3sg avoir argent beaucoup
c'est pourquoi filles daoy
«Il a beaucoup d'argent, c'est pourquoi les filles sont folles de lui/lui courent après »

Dans les exemples (45) et (46), la présence de daoy Y redéfinit l'interprétation de X: dans (45), il introduit un terme (« je ») qui se voit insulter alors qu'a priori il n'est pas concerné par la querelle ; dans (46) Y signifie que l'événement X varie selon les endroits.

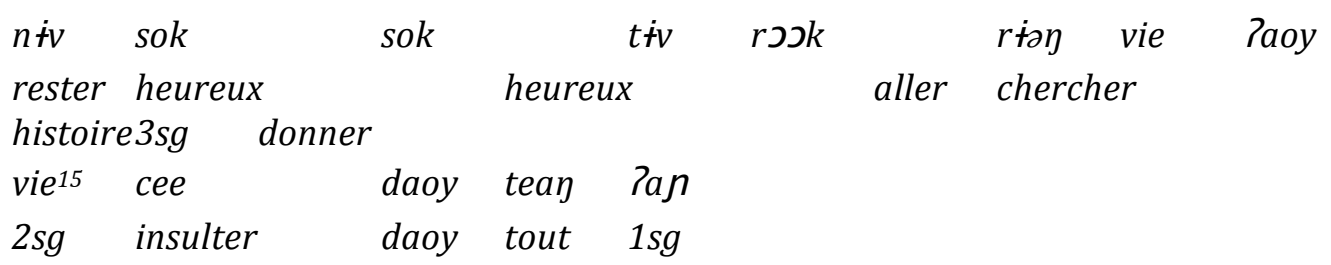

« Tout allait bien mais tu lui as cherché des histoires. Et elle t'a insulté et moi aussi du même coup (alors que je n'y étais pour rien) »

$$
\text { tyay nih mien pliey tleak daoy kanlaey }
$$

\footnotetext{
14 Pour une description systématique de daoy, cf. Paillard [2013a].

${ }^{15}$ Le pronom vie peut désigner aussi bien la $3^{\text {ème }}$ ( $1^{\text {ère }}$ occurrence) que la $2^{\text {ème }}$ personne (2 $2^{\text {ème }}$ occurrence).
} 
jour ce avoir pluie tomberdaoy endroit "Aujourd'hui il pleut à certains endroits »

La deuxième série d'exemples correspond aux cas où, selon Headley, daoy sert à former des adverbes de manière.

$\begin{array}{llll}\text { koat tvaə riły nih daoy cesta?naa } \\ 3 s g \text { faire histoire } & \text { ce daoy intention }\end{array}$

« Il a fait ça de façon délibérée»

(48) knom cay ni7yiey daoy laek

$1 s g$ désire parler daoy privé

«Je voudrais en parler en privé »

Pour cerner le statut de la détermination introduite par daoy, il est intéressant de comparer les exemples avec daoy et ceux où la séquence adverbiale est introduite par yaaj « manière » :

koat baan bat $\quad$ muk pii sallapa? daoy Paatkambay
3sg obtenir disparaître face de art daoy mystère
"La raison pour laquelle il a disparu du monde de l'art est un mystère »

"La raison pour laquelle il a disparu du monde de l'art est un mystère »

(49b) koat baan bat muk pii səllopa? yaay Taatkambay

3sg obtenirdisparaître face de art yaan mystère

" Il a disparu de façon mystérieuse du monde de l'art »

(50a) koat ni7yiey daoy sruəl

3 sg parler daoy facile

«Il parle de manière non conflictuelle/de façon à éviter les conflits »

(50b) koat ni7yiey yaan srual

$3 s g$ parler yaan facile

«Il parle avec (beaucoup de) facilité »

Les séquences avec yaaך peuvent effectivement être caractérisées comme des locutions adverbiales spécifiant le mode de réalisation du procès correspondant à $\mathrm{X}$. En revanche, les séquences avec daoy se présentent comme une détermination externe, sans statut dans X. Cela est confirmé par le fait qu'avec yaay il est possible d'avoir la négation, qui est bloquée avec daoy (on retrouve ici la contrainte évoquée ci-dessus dans le cadre de la comparaison daoy/taam, cf. ex. (43a-c)) ${ }^{16}$.

Dans la dernière série d'exemples, $\mathrm{X}$ et $\mathrm{Y}$ sont des propositions, ce qui correspond aux emplois où, selon Headley, daoy est une conjonction. Nous distinguerons deux grands cas.

\footnotetext{
${ }^{16} \mathrm{~A}$ noter que dans les exemples (47)-(48), yaał est bloqué. On trouve également de nombreux cas où seul yaay est possible, cf. Paillard [2013b].
} 
Dans les données relevant du premier cas, daoy est associé à la particule kaa et est postposé à la séquence correspondant à Y. La particule kaa signifie que la valeur du prédicat n'est pas celle attendue/souhaitée ${ }^{17}$ :

\begin{tabular}{|c|c|c|c|c|c|c|c|c|}
\hline $\begin{array}{l}\text { tvay tbat } \\
\text { bien que }\end{array}$ & $\begin{array}{l}\text { tae koat } \\
\text { restrict } 3 s g\end{array}$ & $\begin{array}{l}\text { baan } \\
\text { obtenil }\end{array}$ & $\begin{array}{l}\text { dəך } \\
\text { savoir }\end{array}$ & $\begin{array}{l}\text { thaa } \\
\text { dire }\end{array}$ & $\begin{array}{l}\text { ræ̇y } \\
\text { histoire }\end{array}$ & $\begin{array}{l}\text { nih } \\
\text { ece }\end{array}$ & $\begin{array}{l}n \dot{+} \eta \\
\text { part }\end{array}$ & $\begin{array}{l}t \dot{+} \dot{v} \\
\text { aller }\end{array}$ \\
\hline doocneh & kaadaoy & tae & koat & kmien & & cuтrəə & & 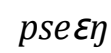 \\
\hline
\end{tabular}

$\begin{array}{lllll}\text { tlay ponman } & \text { kaa1 daoy } & \text { kaa2 knom təəл vie } \\ \text { cher combien } & \text { kaadaoy } & \text { kaa } 1 \mathrm{sg} \text { acheter } 3 \mathrm{sg}\end{array}$

"Même si c'est (très) cher, je vais quand même18 l'acheter »

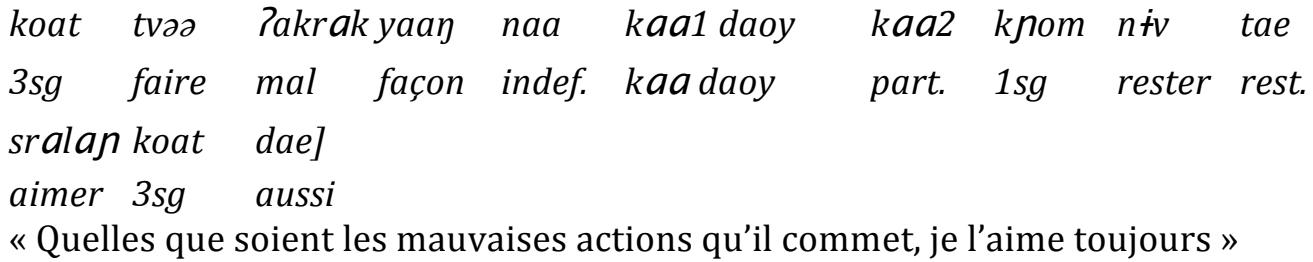

Les trois exemples ci-dessus ont une valeur concessive (Y n'est pas l'obstacle qu'il aurait pu être pour ce qui est de la validation de X). Dans (52), on a la présence du marqueur «bien que». On peut expliciter le mécanisme en jeu dans (53) (que l'on retrouve dans (54)) : le prix élevé de l'objet n'est pas une bonne chose (kaa1), mais il est pris comme repère pour ce qui est de la décision de l'acheter, décision qui, compte tenu du prix, n'aurait pas dû se faire (kaa2).

Dans la seconde série d'exemples, daoy Y définit les conditions dans lesquelles l'événement X a lieu :

$$
\begin{aligned}
& \text { knom saphay (treek haa/khəך/pirbaakcat) nah daoy bay mosk } \\
& 1 \mathrm{sg} \text { heureux(content / furieux / malheureux) très daoy frèreaîné venir } \\
& \text { leey pteah knom } \\
& \text { jouer maison } 1 \mathrm{sg} \\
& \text { "Je suis très heureux (content/furieux/malheureux) que vous veniez me rendre visite » }
\end{aligned}
$$

(56) vie min prosm tiv saalaa daoy yəok leeh thaa kdav kluən 3sg nég. accepter aller école daoy prendre prétextedire chaud corps «Il ne veut pas aller à l'école prétextant qu'il a de la fièvre »

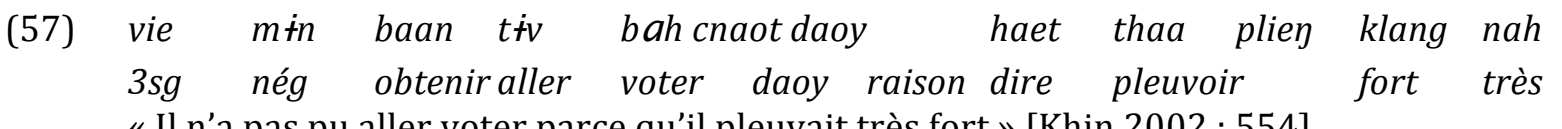
«Il n'a pas pu aller voter parce qu'il pleuvait très fort » [Khin 2002 : 554]

\footnotetext{
17 Sur kaa, cf. Hayman [2011] et Thach [2013: 326 - 341].

18 Le second kaa signifie que « acheter l'objet » n'est pas a priori la bonne valeur (compte tenu du prix). Le mécanisme est le même dans (54).
} 


\begin{tabular}{|c|c|c|c|c|c|c|c|c|}
\hline & kluən & $c a h$ & peek & koat & $k a a$ & samrac cət & $m \dot{t n}$ & $\begin{array}{l}\text { bantaa } \\
\text { continuer }\end{array}$ \\
\hline daoy & corps & vieux & trop & $3 s g$ & kaa & décider & nég. & continuer \\
\hline $\begin{array}{l}\text { kaayie } \\
\text { travail }\end{array}$ & robah & koat $^{19}$ & & & & & & \\
\hline
\end{tabular}

Synthèse : cette présentation des principales valeurs de daoy montre que ce marqueur échappe à toute catégorisation, alors même qu'il occupe une place importante dans la grammaire du khmer. Il est intéressant de noter que les principales grammaires du khmer (Khin [2002], Hayman [2011]), construites en référence aux grammaires des langues indo-européennes, ne mentionnent daoy que de façon ponctuelle.

\section{Conclusion}

Dans le prolongement de cette présentation de quatre unités du khmer, nous voudrions insister sur deux points :

La problématique de l'invariance en tant que fondant l'identité sémantique d'une unité permet de décrire les différentes valeurs de cette unité sans prendre en compte la distinction entre emplois dits lexicaux et emplois dits grammaticaux. Elle évite le recours (abusif concernant une langue comme le khmer) aux notions de grammaticalisation. L'identité d'une unité est indissociable de sa variation ou, pour reprendre une formule de Sarah De Vogüé [2013 $]^{20}$ :

Dans la théorie de l'invariance, il n'y a pas de valeur première qui ensuite varie. C'est la variation qui est première : l'identité est variation.

Concernant les catégories grammaticales, leur association à l'obligatoire qui suppose une distinction stricte entre unités lexicales et unités grammaticales n'est pas fondée, sauf à conférer aux catégories d'une langue le statut de catégories «modèles » ${ }^{21}$. Nous nous référons ici à la définition d'une catégorie proposée par A. Culioli [1999 : 164] qui articule invariance et variation ${ }^{22}$ :

Une catégorie est le produit d'une mise en correspondance de représentations de niveau I23, d'un côté, et de marqueurs de niveau II, de l'autre (ces marqueurs sont

\footnotetext{
${ }^{19}$ Avec daoy Y en tête, la présence de kaa dans X est requise.

${ }^{20}$ Sur l'invariance, cf. également De Vogüé [2006].

${ }^{21}$ A titre d'exemple, on peut citer la manière dont la distinction Imperfectif/Perfectif théorisée par B. Comrie dans son ouvrage Aspect [1976] est réinvestie dans les grammaires des langues d'Asie du Sud-Est (et au-delà !), au détriment des constructions propres à ces langues (sur ce point, cf. Paillard [2013c]).

${ }^{22}$ Sur la notion de catégorie, cf. également Paillard [2013a].

${ }^{23}$ Culioli distingue trois niveaux de représentation: le niveau 1 est celui des opérations mentales (cognition et affect), en tant que telles inaccessibles, le niveau 2 est celui des formes, le niveau 3 celui des représentations métalinguistiques associées. Le niveau 2 (celui des formes) occupe une position charnière. D'un côté, il est en relation avec le niveau 1 : les formes linguistiques sont les traces de l'activité de représentation du niveau 1 ; de l'autre, elles sont en relation avec le niveau 3, celui des représentations métalinguistiques : elles ont alors le statut de marqueurs. Les formes de la langue ont donc un double
} statut:

Cognition-affect traces FORMES marqueurs représentations

niveau $1 \quad$ niveau 2 niveau 3


disposés en réseau, propres à une langue donnée : on aura donc un jeu spécifique de marqueurs auxquels on associe un réseau de valeurs). On obtient ainsi une double relation, non symétrique entre des notions (que pour aller vite, nous appellerons grammaticales) et des marqueurs. Les notions ont des propriétés formelles invariantes et elles permettent donc par sélection et combinaison des regroupements de propriétés ; ces regroupements sont variables et fournissent une multiplicité de chemins possibles entre le niveau I et le niveau II [...]. On voit clairement que le chemin de I à II associe l'invariance et la variation. [...] Une catégorie est toujours une catégorisation.

Dans cette perspective une distinction stricte entre lexique et grammaire ne saurait être maintenue. Selon les marqueurs en jeu, chaque langue compte tenu des formes en jeu peut être considérée comme constituant une réalisation particulière de telle ou telle catégorie. Prendre en compte la très grande diversité des réalisations possibles contribue en retour à un enrichissement de la réflexion sur le statut des catégories. D'où un double mouvement: des catégories vers les formes/les marqueurs, d'une part, des formes/marqueurs dans une langue donnée vers les catégories, d'autre part. Et comme l'écrit encore Culioli [ibidem : 163] :

Le concept de marqueur, tel qu'il a été défini plus haut, exclut toute séparation radicale entre lexique et grammaire. Il n'existe pas de catégorie grammaticale sans composante lexicale, de même qu'il n'existe pas de lexique qui ne comporte pas de propriétés formelles d'ordre grammatical. Bref, toute grammaire est grammaire lexicale.

\section{Bibliographie}

BISANG Walter, 2011, "Grammaticalization and the areal factor in East and mainland Southeast Asian languages"

accessible sur http://www.linguistik.unimainz.de/bisang/publications/

ComriE Bernard, 1976, Aspect, Cambridge: Cambridge Textbooks.

Culioli Antoine, 1999, "Les modalités d'expression de la temporalité sont-elles révélatrices de spécificités culturelles?», Interfaces 5, repris dans Pour une linguistique de l'énonciation T. II, Paris : Ophrys, 159-178.

DE VoGuË Sarah, 1995, "L'effet aoristique », in BOUSCAREN Janine, FRANCKEL Jean-Jacques \& RoBert Stéphane (éds), Langues et langage, Paris : PUF, 247-261.

DE VoguË Sarah, 2006, "L'invariance culiolienne », in : DuCARD Dominique \& NoRmand Claudine (éds), Antoine Culioli. Un homme dans le langage, Paris : Ophrys, 302-332.

DE VoGUË Sarah, 2013, "Invariance contre grammaticalisation : à propos des variations dans le champ de la condition », Langages ${ }^{\circ} 190$, Paris : Armand Colin, 91-99.

Dictionnaire Cambodgien - Français, 2007, Tomes I et II, Phnom Penh.

ENFIELD Nick, 2003, Linguistic Epidemiology, London: Routledge.

ENFIELD Nick, 2004, "Areal grammaticalization of postverbal "acquire" in Mainland and Southeast Asia », in Papers from the Eleventh Annual Meeting of SEALS, Tempe: Arizona State University, 275-296.

HaYman John, 2011, Cambodian Khmer, Amsterdam/Philadelphie, « London Oriental and African Language Library » 16: John Benjamins Publishing Company. 
Headley Robert, 1977, Cambodian English Dictionary, Washington D.C.: The Catholic University of America Press.

Heine Bernd \& KuTEva Tatiana, 2002, World Lexicon of Grammaticalization, Cambridge: Cambridge University Press.

JaKobSon Roman, 1967, Essais de linguistique générale, Paris, Editions de Minuit.

JEnNER Philip, 2009a, A Dictionary of pre-Angkorian Khmer (edited by Doug Cooper), Canberra, "Pacific Linguistics", PL 597: ANU.

JENnER Philip, 2009b, A Dictionary of Angkorian Khmer (edited by Doug Cooper), Canberra, "Pacific Linguistics" PL 598: ANU.

JEnny Mathias, 2005, The Verb System of Mon, Universität Zürich: Arbeiten des Seminars für Allgemeine Schprachwissenschaft $N^{\circ} 19$.

KHIN Sok, 2002, La grammaire du khmer moderne, Paris : Editions You-Feng.

Paillard Denis \& THACH Deth, 2009, "A propos de trav en khmer contemporain", Cahiers de linguistique d'Asie orientale, $\mathrm{n}^{\circ} 38 / 1$, Paris : EHESS, 71-124.

PaIllard Denis, 2011, "About 7aoy "give" in contemporary Khmer", Mon Khmer Studies Journal, Special issue $\mathrm{n}^{\circ}$ 2, Austroasiatic studies, Papers from ICAAL4, 124-138.

PaIllard Denis, 2013a, "Les marqueurs discursifs comme catégorie », in DuFAYE, Lionel \& Gournay, Lucie (éds), Benveniste après un demi-siècle. Regards sur l'énonciation aujourd'hui, Paris : Ophrys, 154-181.

Paillard Denis, 2013b, "About polyfunctionality in Khmer: the case of daoy", Communication présentée à SEALS 23, Bangkok, 28-30 mai 2013.

PaIllard Denis, 2013c, "Les constructions verbales en série en khmer contemporain », Faits de Langues 41, Berne : Peter Lang, 11-40.

TнAсн Deth, 2009, "trav et la diathèse passive en khmer », Faits de langues. Les Cahiers 2, Paris/Gap : Ophrys, 128-175. 